\title{
"DISEÑO AMBIENTALMENTE CONSCIENTE (DAC). HACIA UNA ARQUITECTURA SUSTENTABLE PARA EL HOMBRE Y LA SOCIEDAD". INNOUACIÓN PEDAGÓGICA IMPLEMENTADA EN LA CÁTEDRA ARQUITECTURA II UPB
}

\footnotetext{
María Laura BOUTET, / Juan C. VIRILI
}

Jefa de Trabajos Prácticos; Profesor adjunto a cargo, Cátedra Arquitectura II Unidad Pedagógica "B", Área del Diseño, Facultad de Arquitectura y Urbanismo, UNNE

Palabras Clave: Proceso proyectual, Adecuación climática, Estrategia didáctica.

Keywords: Design process, climate adequacy, didactic strategy.

\section{RESUMEN}

Se presenta una experiencia de innovación pedagógica implementada en la cátedra Arquitectura II - UP "B", referida a la introducción del Diseño Ambientalmente Consciente en la resolución de problemas de diseño en el Taller de Arquitectura. Se analizan los resultados obtenidos y sus implicancias, en diferentes dimensiones de interés en la práctica docente universitaria, contrastadas con encuestas realizadas a los alumnos de la asignatura. La sistematización y valoración de las actividades desarrolladas permite calificar la experiencia como muy satisfactoria, no solo desde el punto de vista disciplinar, sino también por propiciar una actitud crítica y reflexiva frente al desafío de la Arquitectura Sustentable.

\section{ABSTRACT}

The chair Arquitectura II UP B presents an experience of pedagogic innovation. It refers to the introduction of Environmentally Conscious Design in solving design problems in the Architecture Workshop. The obtained results and his implications are analyzed, in different dimensions of interest in the educational university practice, confirmed by surveys realized to the students of the subject. The systematizing and valuation of the developed activities can be qualified as very satisfying experience, not only from the point of view of discipline, but also for propitiating a critical and reflexive attitude opposite to the challenge of the Sustainable Architecture. 
"recuperación de conocimientos previos e integración de contenidos, predisposición para el trabajo grupal colaborativo como modo de abordar la masividad y grado de aprendizaje de los contenidos disciplinares"...

\section{OBJETIVOS}

- Exponer aportes didácticos a partir de una experiencia de innovación pedagógica implementada en la asignatura Arquitectura II, UP "B", referida a la introducción del "Diseño Ambientalmente Consciente" en la resolución de problemas de diseño en el TaIler de Arquitectura.

- Analizar sus implicancias relativas a diferentes dimensiones, desde del método de proyectos como enfoque pedagógico: apreciación sobre la metodología de trabajo, motivación, desarrollo de la capacidad creativa y del pensamiento crítico e intuitivo, recuperación de conocimientos previos e integración de contenidos, predisposición para el trabajo grupal colaborativo como modo de abordar la masividad y grado de aprendizaje de los contenidos disciplinares. Evaluación.

- Identificar los puntos críticos de las estrategias didácticas aplicadas, sobre los cuales se debe intervenir, y enunciar nuevas líneas de acción.

\section{INTRODUCCIÓN}

La Arquitectura Sustentable, conocida también como Arquitectura Sostenible, Arquitectura Verde, Ecoarquitectura o Arquitectura Bioclimática, es un modo de concebir la arquitectura buscando aprovechar los recursos naturales a fin de minimizar el impacto ambiental de las construcciones sobre el ambiente natural y sobre los habitantes. El Diseño Ambientalmente Consciente (DAC), que es una nueva concepción más amplia, difundida en los últimos años a nivel internacional y latinoamericano, es el método por el cual se puede alcanzar una Arquitectura Sustentable (CZAJKOWSKI Y GÓMEZ, 2007).

Estos conceptos han tomado preponderancia a partir del colapso de las fuentes convencionales de generación de energía. Frente a esta grave situación, los futuros arquitectos y los profesionales tenemos una gran responsabilidad en reducir al mínimo las consecuencias negativas para el medio ambiente, mediante un diseño eficaz de los edificios, que modere el consumo energético aprovechando las fuentes de energías renovables (como la solar), con disposiciones de diseño puramente arquitectónicas (pasivas), que favorezcan al bienestar de los usuarios, sin necesidad de recurrir -o por lo menos minimizando su uso- a medios artificiales de climatización.

El término arquitectura sustentable es una derivación del término desarrollo sustentable: "EI desarrollo es sustentable cuando satisface las necesidades de la presente generación sin comprometer la capacidad de las futuras generaciones para que satisfagan sus propias necesidades" ONU (1987). Esta definición clave proporciona un punto de partida para analizar sustentabilidad en el ambiente construido, que abarca tres dimensiones fundamentales: ambiental, económica y social. Se considera que un proyecto es exitoso cuando logra un equilibrio entre las tres.

Uno de los criterios que se debe tener en cuenta para comenzar a hablar de sustentabilidad es poner cuidado en las fases, escalas y procesos de la arquitectura. Es prioritario focalizarse en el diseño, pues es en las fases iniciales del proyecto cuando se pueden tomar las decisiones más trascendentes y de mayor impacto ambiental positivo, con mayor libertad y a costo cero. Por ello, el estudiante de arquitectura debe poseer conocimientos básicos y generales sobre el Diseño Ambientalmente Consciente desde los primeros años de la carrera, para afrontar con soltura la fase de ideación de un edificio, manejando estrategias respetuosas con el medio ambiente a fin de que el resultado sea exitoso desde todo punto de vista (ambiental, económico y social).

Con estos lineamientos, y sumado al manifiesto interés de la cátedra en el abordaje de la relación 
clima y arquitectura, fue introducido dicho eje temático en el Ciclo Lectivo 2011. La Arquitectura Sustentable constituye además una línea prioritaria de investigación y desarrollo de la FAU - UNNE, de lo que se desprende la pertinencia de la incorporación formal de esta unidad temática al programa de la asignatura, con fundamentos técnicos y científicos, como transferencia a la docencia de la formación específica de posgrado de los autores. Son sus objetivos principales:

- Que el alumno logre el desarrollo de una postura reflexiva ante la situación energética edilicia para resolver en forma adecuada la relación Clima, Hombre y Arquitectura, mediante el análisis crítico de obras reconocidas a nivel local, nacional o internacional, en las que se verifiquen disposiciones arquitectónicas respetuosas con el medio ambiente. - Que el alumno logre la aplicación creativa de Estrategias de Diseño Ambientalmente Consciente (DAC) expresadas en la idea generadora del proyecto, a partir del conocimiento de las condicionantes climáticas locales.

Los resultados logrados fueron muy satisfactorios, ya que los alumnos pudieron incorporar a su repertorio de conocimientos conceptos básicos de transferencia de calor, clima, asoleamiento, entre otros, aplicándolos en un producto original: el Proyecto de un "Centro de Formación e Innovación Educativa", en el ciclo lectivo 2011, y el Proyecto de un "Centro de Capacitación para Jóvenes Emprendedores", en el ciclo lectivo 2012.

\section{DESARROLLO}

Se describen a continuación las actividades, contenidos, aplicación práctica y enfoque pedagógico de la experiencia.

Actividades de la unidad. Las actividades están diseñadas con el objetivo de promover una partici- pación activa de los alumnos en la construcción del conocimiento y de la metodología de diseño arquitectónico. A tal fin, se plantean dos bloques teórico-prácticos, con apoyatura de clases expositivas, con la utilización de material visual para mejor comprensión del tema y con instancias de participación de los alumnos. El primer bloque es introductorio, y culmina con un esquicio individual/grupal. Los resultados del esquicio permiten verificar los conocimientos previos de los alumnos en relación con las temáticas abordadas, y la ejercitación de propuestas de diseño ambientalmente consciente a nivel intuitivo. El segundo bloque abarca los contenidos fundamentales de la unidad temática y culmina con el desarrollo de un ejercicio práctico de diseño: un proyecto de un edificio de media complejidad, aplicando estrategias DAC. El repertorio de temas varía en cada ciclo lectivo, siempre tratándose en este caso de trabajar con edificios de media complejidad, a fin de que los alumnos logren poner en juego las estrategias de diseño en estudio, sin mayores complicaciones en el aspecto funcional, y lleguen a un resultado a nivel de partido arquitectónico avanzado.

Contenidos de la Unidad. Se brinda una introducción a los conceptos de Diseño Ambientalmente Consiente (DAC) y Arquitectura Sustentable, en el contexto de crisis energética y de agotamiento de recursos no renovables, destacando el rol fundamental del arquitecto en la realización de una arquitectura respetuosa del medio ambiente. Se analizan ejemplos de arquitectura catalogada como "sustentable", a fin de discutir la pertinencia de esta calificación tan difundida. Se aborda la relación Clima, Hombre y Arquitectura, valorando la influencia de los elementos y factores del clima, en las condiciones de la "envolvente" como mediadora entre el clima exterior y los ambientes interiores. Se brindan ejemplos de Arquitectura Vernácula, como máxima expresión de estos conceptos. Se concibe la Radiación Solar como 
principal elemento del clima y fuente de energía renovable por excelencia, subrayando que en un clima riguroso como el de la Región NEA es el principal condicionante de la obra arquitectónica. Se introducen contenidos de geometría solar para un estudio más profundo del asoleamiento y dimensionado de protecciones solares. Además se enuncian las formas básicas de transferencia de calor a través de la envolvente arquitectónica, por conducción, convección y radiación, como así también el concepto de confort higrotérmico. En relación con ello, se brinda un panorama del marco normativo referido al acondicionamiento térmico y eficiencia energética de los edificios, a partir de las Normas del Instituto Argentino de Normalización y Certificación (IRAM) y las nuevas normas nacionales de Certificación Energética. Se destacan iniciativas que se están implementando en nuestro país, impulsadas por diferentes centros y grupos de investigación.

Con esta base teórica, se analizan algunas Estrategias de Diseño Ambientalmente Consciente (DAC), que a los fines del ejercicio práctico que se desarrollará en taller, se han clasificado en seis categorías básicas: 1) implantación y orientación; 2) forma de la tipología y distribución interna; 3) tecnología de la envolvente constructiva; 4) aberturas y vidriados; 5) protecciones solares y 6) ventilación natural. En todos los casos se brindan ejemplos de obras construidas y se proporciona a los alumnos una guía didáctica que detalla las estrategias de diseño ambientalmente consciente, correspondientes a cada categoría (BOUTET Y VIRILI, 2012).

Aplicación práctica. El ejercicio se organiza en dos etapas: una etapa de análisis, en la que se desarrolla una tarea de investigación y análisis crítico, de corta duración para la apropiación de las estrategias en estudio; una etapa de diseño creativo, con el objetivo básico de alcanzar un diseño respetuoso del medio ambiente y acorde con las con- dicionantes climáticas del sitio de emplazamiento, aplicando una o varias estrategias DAC. El proceso de desarrollo del ejercicio tiene varios cortes o paréntesis en los que se realizan actividades de exposición, puesta en común y esquicios como disparadores de las distintas etapas. Todo lo expuesto se ejecuta en un tiempo aproximado de dos meses, y la modalidad de trabajo es en grupos de tres a cuatro integrantes, reunidos en comisiones coordinadas por los docentes, en el Taller de Arquitectura.

Enfoque pedagógico. La experiencia didáctica descrita se basa en la enseñanza indirecta y el aprendizaje por descubrimiento, encuadrándose en el "Método de Proyectos", definido por COLS, E. (2008) como el "conjunto de actividades orientadas hacia una realización o producción concreta". Emerge de una visión de la educación en la cual los estudiantes toman una mayor responsabilidad de su propio aprendizaje y en donde aplican, en proyectos reales, las habilidades y conocimientos adquiridos en el aula, con un mayor entendimiento del rol tan importante que tienen en la sociedad. Envuelve entre otros aspectos los siguientes: 1) un planteamiento que se basa en un problema real y que involucra distintas áreas; 2) oportunidades para que los estudiantes realicen investigaciones que les permitan aprender nuevos conceptos, aplicar la información y representar su conocimiento de diversas formas; 3) colaboración entre los estudiantes, docentes y otras personas involucradas con el fin de que el conocimiento sea compartido y distribuido entre los miembros de la "comunidad de aprendizaje"; 4) el uso de herramientas cognitivas y ambientes de aprendizaje que motiven al estudiante a representar sus ideas, como laboratorios, hipermedios, computacionales, aplicaciones gráficas y otros.

Este método es un modelo innovador de enseñanza-aprendizaje, que supone la definición de nuevos roles para el alumno y para el profesor. Aquí el aprendizaje pasa de las manos del profesor a las 
del alumno, de tal manera que este pueda hacerse cargo de su propio aprendizaje. El profesor deja de pensar que tiene que hacerlo todo, y da a sus alumnos la parte más importante; se vuelve estudiante al aprender cómo los alumnos aprenden, lo que le permite determinar cuál es la mejor manera en que puede facilitarles el aprendizaje, como un asesor o colega, más que como un experto.

Este es, naturalmente, el modo de trabajo en el Taller de Arquitectura. Es el ámbito propicio para aprender haciendo (SCHON, 1992), donde el alumno asume el papel de profesional que debe defender su proyecto, y donde el docente no es solo un transmisor de conocimientos, sino que asume el papel de facilitador de los procesos de los alumnos y del desarrollo de las actividades.

En función de lo expresado precedentemente, desde el enfoque pedagógico del Método de Proyectos, la experiencia didáctica descrita implica la resolución de un problema real, en un contexto físico y socio-cultural también real, para lo cual los alumnos deben movilizar un conjunto de saberes y actividades previas (clases expositivas, charlas con especialistas, estudio de casos o análisis de modelos, análisis bibliográfico), en forma cooperativa (aprendizaje colaborativo) y tomando decisiones de manera ágil, para llegar a un producto concreto.

A continuación se conceptualizan brevemente las categorías de análisis didáctico-pedagógico que se mencionan en los objetivos, definidas a fin de verificar los resultados de la experiencia y detectar los puntos críticos que intervenir.

Apreciación sobre la metodología de trabajo. Los proyectos pueden tener múltiples productos (preliminares, finales, realizados individualmente o en grupo). Los productos pueden ser organizados por etapas, que proveen puntos específicos de control, con los que tanto los docentes como los alumnos pueden valorar el avance, hacer cambios de dirección y estimar tiempos reales para la ter- minación del proyecto. Los productos terminados dan la oportunidad al alumno de demostrar su aprendizaje. Las actividades deben ser construidas en bloques, de manera que lleven a los alumnos a alcanzar contenidos de conocimiento, de desarroIlo de habilidades y de resultados de procesos. Al estar centrado en el alumno, el método de proyectos ocasiona que se sienta más motivado pues él es quien resuelve los problemas, planea y dirige su propio proyecto; se convierte en un descubridor, integrador y presentador de ideas; se muestre comunicativo, afectuoso, productivo y responsable; use la tecnología para manejar sus presentaciones - ampliar sus capacidades; trabaje colaborativamente con otros; construya, contribuya y sintetice información; encuentre conexiones interdisciplinarias entre ideas; se enfrente a obstáculos, busque recursos y resuelva los retos que se le presentan.

Motivación. La diversificación de la tarea en el ejercicio de taller que se plantea, y de los modos de participación de los estudiantes, para investigar, indagar, crear, diseñar, permite crear un clima en el cual los estudiantes pueden practicar una variedad de habilidades y disposiciones para aprender a aprender. Ayuda a los estudiantes a desarrollar la iniciativa propia, la persistencia y la autonomía. Invita a los estudiantes a explicar o defender su posición ante los demás en sus proyectos grupales, para que su aprendizaje sea personal y puedan valorizarlo y, por lo tanto, se sienta más motivado.

Desarrollo de la capacidad creativa y del pensamiento crítico e intuitivo. Mediante el temaproblema planteado, sumado a las actividades presenciales que recuperan el conocimiento intuitivo de los estudiantes y profundizan los conceptos aprendidos, se abre el camino para emitir juicios de valor en el proceso de resolución del proyecto, fundamentando sus ideas y promoviendo a la vez el desarrollo de la creatividad. 
"Esto no solo da a los estudiantes la oportunidad de demostrar lo que han aprendido, sino que además puede incrementar la validez y autenticidad de la evaluación del proyecto. La evaluación del aprendizaje de los estudiantes por parte de los docentes puede ser complementada con la evaluación de un colega, del cliente del proyecto y de autoevaluaciones de los estudiantes, como retroalimentación de su desempeño" (ITESM, sf).

Recuperación de conocimientos previos e integración de contenidos. Se pretende que las actividades permitan a los alumnos buscar información para resolver problemas, construir sus propios conocimientos, favoreciendo la retención y transferencia, aplicando las habilidades adquiridas a una variedad de contextos (aprendizaje significativo). El método de proyectos permite diversas aproximaciones al aprendizaje, ya que ofrece múltiples maneras de participar y demostrar los conocimientos: exposiciones en pequeños grupos, en plenarios, esquicios, correcciones sucesivas del proyecto, autoevaluación, entre otras. La diversidad de saberes movilizados y aprendidos durante el proyecto permite integrar conceptos a través de áreas de diferentes materias.

Predisposición para el trabajo grupal colaborativo como modo de abordar la masividad. El profesor que interactúa con cada estudiante o grupo pequeño de estudiantes que están desarrollando un mismo proyecto debe estar en capacidad de administrar el tiempo que le dedica a cada grupo y de reconocer la particularidad de cada propuesta. Esta tarea se ve dificultada por la condición de masividad característica de los primeros años de la carrera, por lo que el método de proyectos, al incentivar el trabajo grupal colaborativo y la autoevaluación, permite al docente actuar como facilitador del aprendizaje de los estudiantes, que se supone se logra en la medida en que puedan resolver exitosamente el proyecto. Así, las condiciones en que este se desarrolla permiten a los alumnos desplegar habilidades de colaboración y negociación, en lugar de competencia, ya que la interdependencia y la colaboración son cruciales para lograr que el proyecto funcione. El docente debe asegurar el trabajo que tendrá que realizar cada participante del grupo.

Grado de aprendizaje de los contenidos disciplinares. Los contenidos manejados en el método de proyectos deben ser significativos y relevantes para el alumno, ya que presentan situaciones y problemáticas reales. En este caso, se aborda en profundidad el Diseño Ambientalmente Consciente como eje central del desarrollo del proyecto de diseño, propiciando la investigación, comprensión y aplicación de estrategias respetuosas con el medio ambiente, en el contexto de crisis energética imperante.

De esta manera, se trabaja en un tópico de actualidad, relevante y de interés local. El método de proyectos tiene relación con una amplia gama de técnicas de enseñanza-aprendizaje, que trabajadas en conjunto, crean un ambiente altamente propicio para la adquisición y el desarrollo no solo de conocimientos disciplinares, sino también de habilidades sociales relacionadas con el trabajo en grupo y la negociación, habilidades profesionales y estrategias propias de la disciplina y preocupación por la relevancia y significación (social, cultural, profesional, personal) de los proyectos.

Evaluación. La presentación de avances del proyecto por parte de los alumnos constituye un recurso para la evaluación; permite al profesor tener diversos elementos para valorar el desarrollo de aquel y los aprendizajes que los alumnos van adquiriendo durante el proceso de diseño. Se recomienda que todos los proyectos tengan una o más presentaciones públicas de avance para evaluar resultados relacionados con el trabajo del proyecto.

Esto no solo da a los estudiantes la oportunidad de demostrar lo que han aprendido, sino que además puede incrementar la validez y autenticidad de la evaluación del proyecto. La evaluación del aprendizaje de los estudiantes por parte de los docentes puede ser complementada con la evaluación de un colega, del cliente del proyecto y de autoevaluaciones de los estudiantes, como retroalimentación de su desempeño (ITESM, sf). 
1a. Exposición de trabajos a cargo de alumnos de la asignatura. 1b. Trabajo en Taller. Esquicio de Ideas Generadoras en Maquetas.

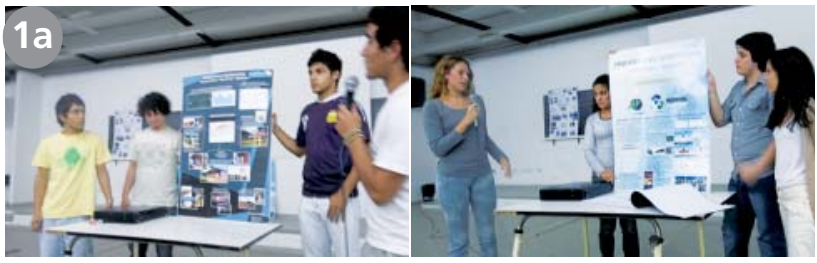

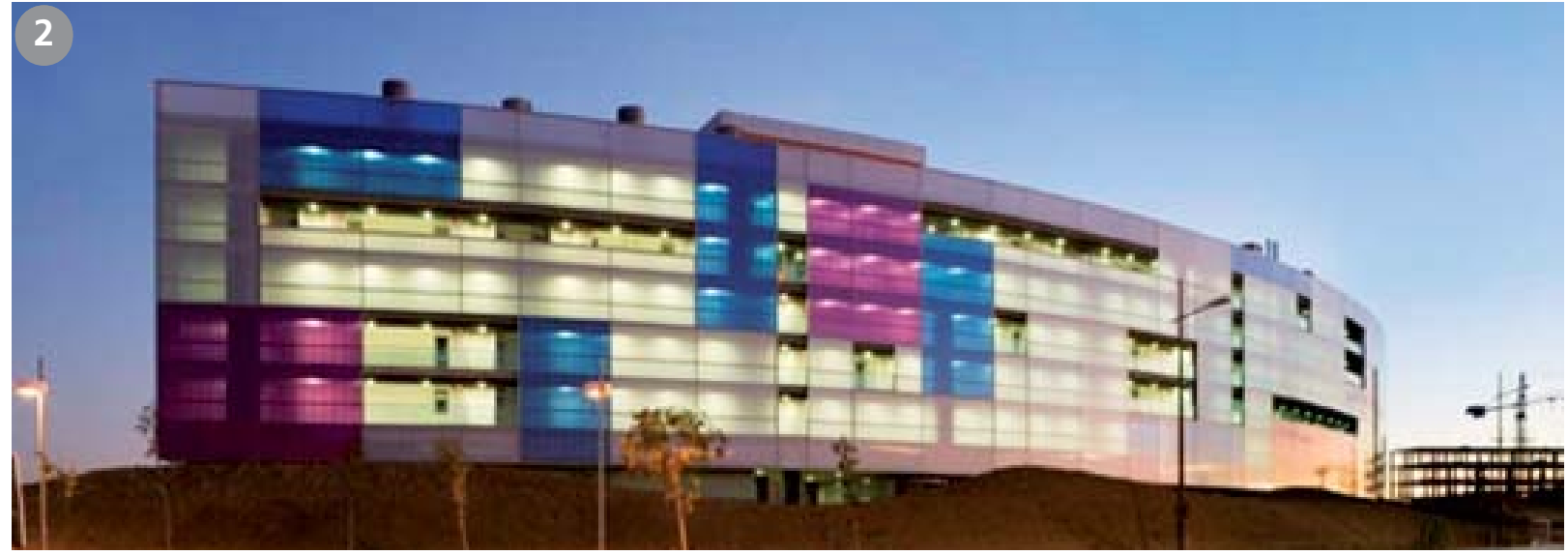

\section{RESULTADOS}

En el presente artículo se presentan los resultados del tema-problema desarrollado en el ciclo lectivo 2012: "Centro de Capacitación para Jóvenes Emprendedores", que corresponde al segundo año de implementación de la propuesta pedagógica.

Dotado de cinco aulas y SUM, con una superficie cubierta aproximada de $600 \mathrm{~m} 2$, como condición básica de un espacio educativo, los alumnos debían tener en cuenta el tratamiento de los espacios pedagógicos (aulas), a fin de lograr adecuadas condiciones de bienestar térmico y lumínico. El proyecto se localizó en un terreno en esquina de la ciudad de Resistencia, elegido por contar con una orientación desfavorable, como desafío para resolver mediante la aplicación de estrategias de Diseño Ambientalmente Consciente (DAC).

En la etapa de análisis, cada grupo de alumnos analizó las estrategias de diseño correspondientes a una categoría que se le asignó, en función de la cual seleccionó una obra en la que se verificara la aplicación de esas estrategias como idea generadora o factor fundamental de diseño, identificando cómo se produce la relación clima-hombre-arquitectura, y plasmando dicho análisis en un panel síntesis. El hecho de que la obra para analizar fuese de libre elección permitió verificar si los alumnos comprendieron verdaderamente la categoría de análisis que se les asignó. A fin de integrar y articular las producciones de todos los grupos y de lograr un conocimiento integral de todas las Estrategias DAC, se realizó una exposición en clase.

Para la realización de la propuesta, los alumnos debían tomar como "idea generadora" las estrategias
2. Trabajo N. 1. Categoría: "Implantación y Orientación" Alumnos: Astori, Agostina; Meza, Matías; Zajarías, Alexis.

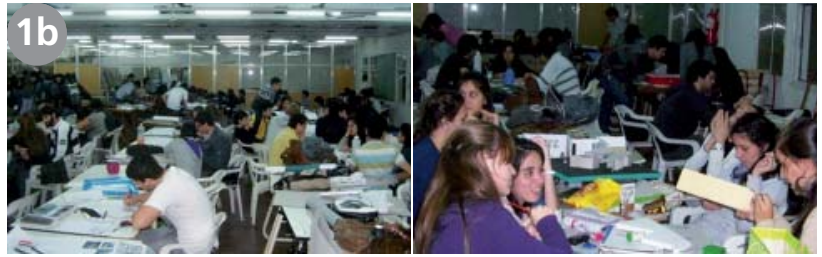

DAC incluidas en la categoría estudiada e identificada en la etapa de análisis. Esta consigna fue resistida al principio, pues los alumnos vieron condicionado su diseño, pero finalmente los ayudó a lograr una buena justificación de su propuesta y a superar el "salto al vacio" frente a las primeras ideas. En ello contribuyó un "Esquicio de Idea Generadora" como disparador de la Etapa de Diseño Creativo en dos jornadas de trabajo en clase a nivel de maqueta de estudio.

A continuación se ilustran las diferentes etapas del ejercicio y se exponen algunos de los trabajos realizados por los alumnos que fueron sistematizados para su análisis. En cada caso se muestra la obra analizada de acuerdo con la categoría asignada a cada grupo, el desarrollo de la idea generadora y el resultado final integrador.(Imágenes 1a y 1b)

Obra analizada: Hemiciclo Solar; Móstoles Sur, Madrid, España; Luis Larrea y asociados. Por su forma, el hemiciclo solar sigue al sol. La envolvente se concibe como un gran captador solar durante el invierno y un inmenso umbráculo en verano mediante celosías. La solución constructiva permite disposiciones flexibles, diferentes niveles de apertura u ocultación. (Imagen 2)

Propuesta de Diseño. Evolución de la idea generadora. Descripción Trabajo N. ${ }^{\circ} 1$. La propuesta es morfológicamente interesante, cumpliendo con todas las estrategias DAC, mediante un partido abierto organizado en paquetes funcionales, dispuestos en forma oblicua al terreno para un mejor aprovechamiento de los recursos naturales del sitio, destacándose en la idea generadora la categoría "Implantación y Orientación". Se plasmó el trabajo de análisis realizado en el tratamiento de la envol- 
3a. Propuesta de Diseño. Evolución de la idea generadora. 3b. Resultado final. 4a. Propuesta de Diseño.

4b. Evolución de la idea generadora. 4c. Resultado final.

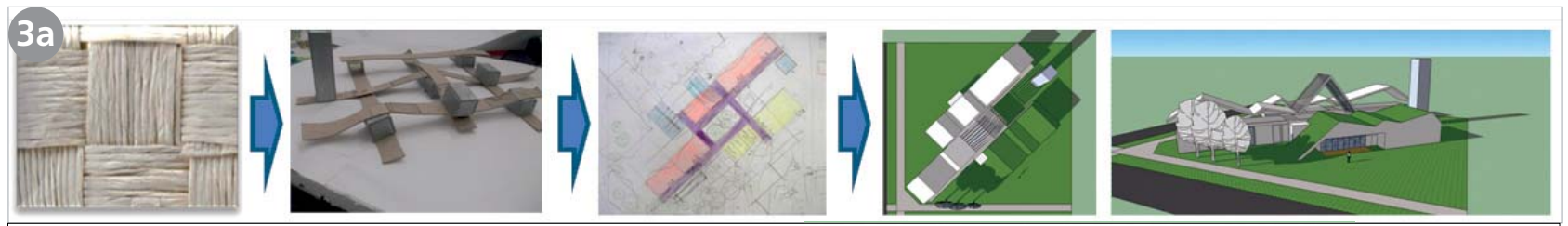

$3 b$
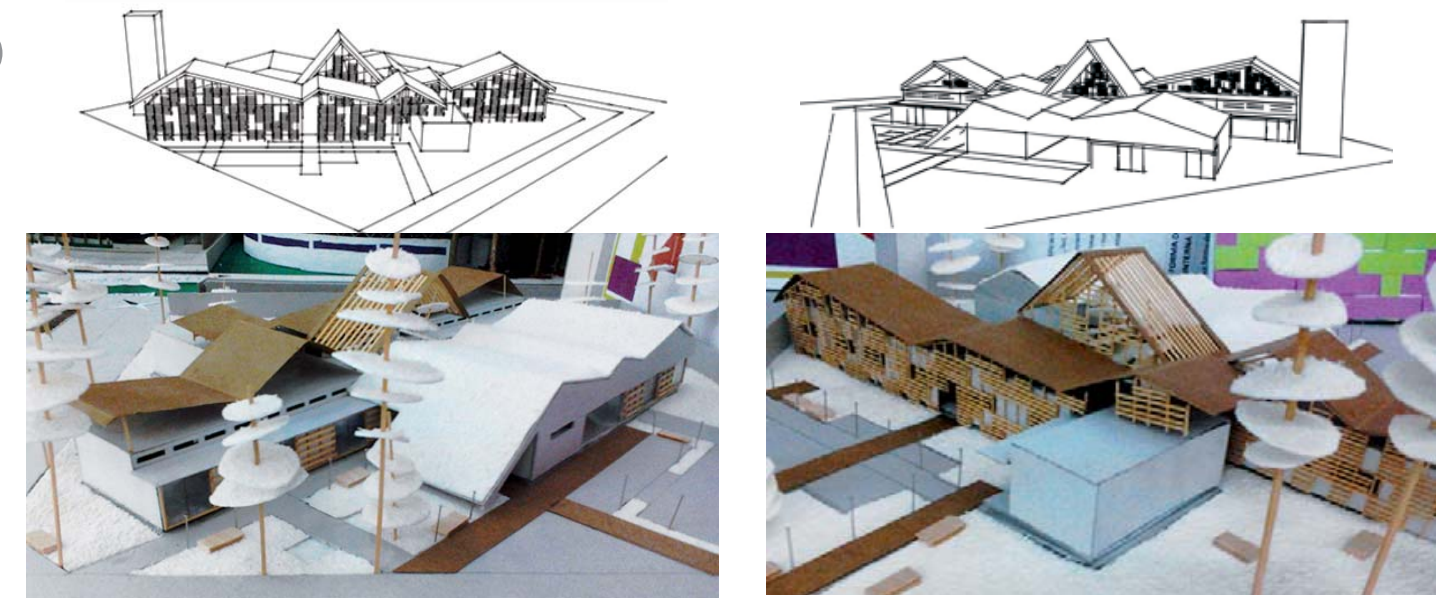

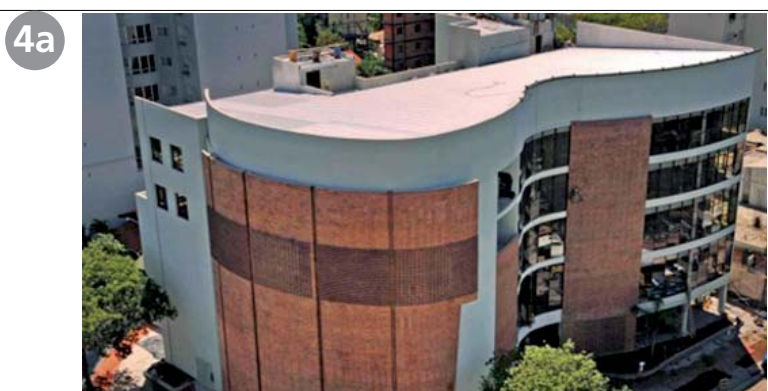

vente arquitectónica mediante recursos como: cubiertas verdes, techos sombras, utilización de materiales regionales, parasoles según orientación, diferenciación de aberturas para aprovechamiento de iluminación y ventilación natural cruzada y por efecto chimenea, forma de la tipología con su eje mayor en dirección este-oeste, disponiendo superficies menores en las orientaciones menos favorables. (Imágenes 3a y 3b).

Trabajo N. ${ }^{\circ}$ 2. Categoría: "Aberturas y Vidriados"

Alumnas: Fernández Yasque, Camila ; Gómez Braccone, Carla; Kolesnik, Eliana. Obra analizada: Casa de las Culturas; Resistencia, Chaco; Instituto de Cultura, Gobierno de la Provincia del Chaco. El lugar de implantación de la obra posee un clima cálido y húmedo, donde la temperatura y la radiación solar alcanzan altos valores casi todo el año, razón por la cual las fachadas vidriadas no son la mejor solución; considerándose hoy en día con un alto valor estético y tecnológico, se evidenció la necesidad de valorar dichas superficies integralmente, partiendo de su papel como filtro de las condiciones climáticas hacia el interior, ya que interactúan con los flujos energéticos naturales que inciden sobre ellas. Propuesta de Diseño. (Imagen 4a). Idea generadora. (Imagen 4b). Resultado final. (Imagen 4c).
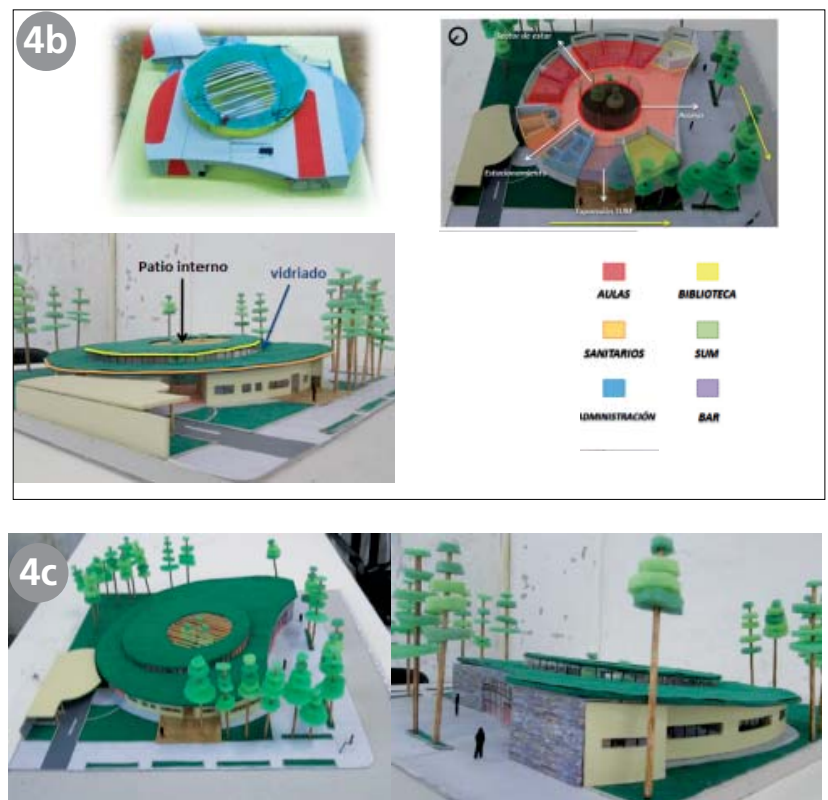

Descripción Trabajo N. ${ }^{\circ}$ 2. Los locales se organizan a partir del patio interno o "pulmón verde", en torno del cual se distribuyen los paquetes funcionales. El patio interno se halla protegido por un pergolado y sobreelevado para favorecer el efecto chimenea y la ventilación cruzada de las aulas. La fachada se resuelve con doble vidriado hermético (DVH): float incoloro $4 \mathrm{~mm}+$ cámara de aire $9 \mathrm{~mm}+$ float stopsol tipo reflectivo y carpinterías de aluminio con burletes elastoméricos. El análisis crítico de la obra seleccionada en la etapa de análisis permitió detectar resoluciones no adecuadas al sitio geográfico de implantación, que se tuvieron en cuenta en el momento de diseñar.

Trabajo N. ${ }^{\circ}$ 3. Categoría: "Ventilación Natural"

Alumnas: González, Evelyn; Montero, Yesica; Morlino, Ma. 
5.Casa Diamante; Diamante, Entre Ríos.

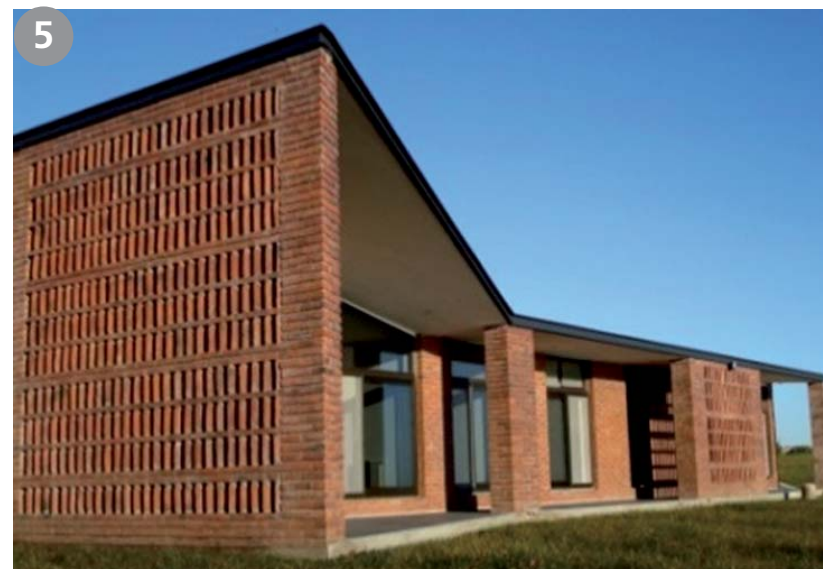

5a. Propuesta de Diseño: idea generadora.

5b. Resultado final.

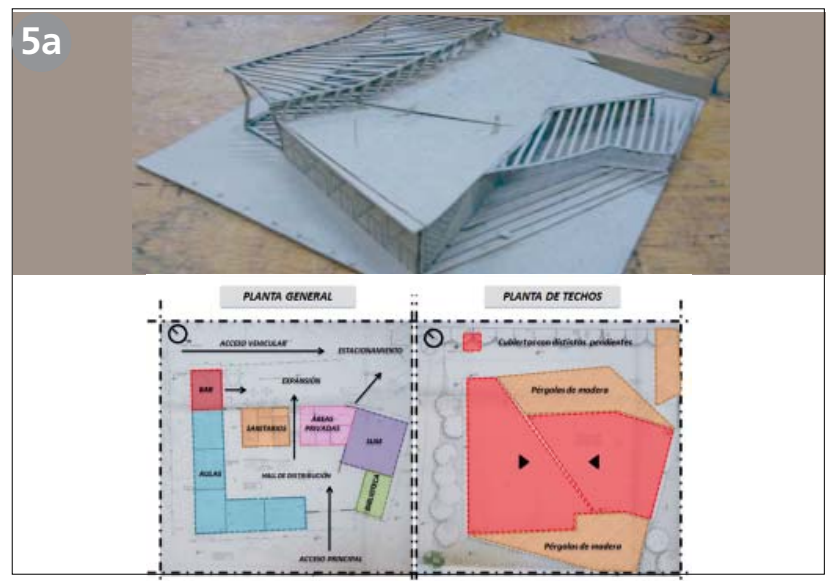

$5 b$

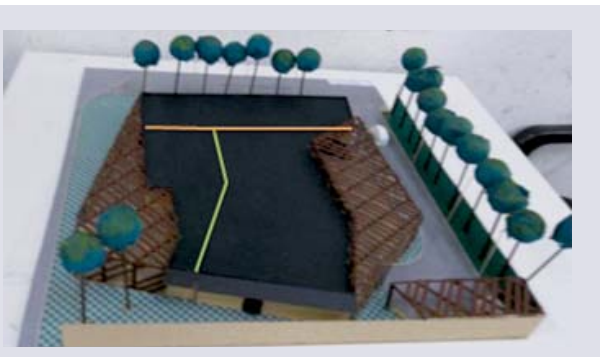

José. Obra analizada: Casa Diamante; Diamante, Entre Ríos; Marsino Arquitectos. Recibe vientos húmedos y predominantes del oeste, por eso se disponen paredes que reducen las fuerzas de aquellos, con una segunda función de protección solar. Las diferentes inclinaciones y ángulos que forman los techos para la distribución de los vientos y las galerías por donde transcurren hasta entrar en la vivienda, brindando confort interior, hacen que pueda definirse a esta obra como un buen ejemplo de ventilación natural. (Imagen 5). Propuesta de Diseño: Idea generadora. (Imagen 5a). Resultado final. (Imagen 5b).

Descripción Trabajo N. 3 . El objetivo fue desarrollar una propuesta que expresara claramente la categoría de diseño ambientalmente consciente que les había tocado, en este caso "Ventilación Natural". Partiendo de la primera etapa de análisis, de la cual se tomaron los conceptos de los quiebres de la cubierta y ventanas altas, se continuó con la complicación que implicaba la implantación en el terreno, siendo este un factor fundamental. Este trabajo tuvo un análisis muy minucioso sobre las corrientes de los vientos en la ciudad de Resistencia. Por esta razón, se priorizó la morfología de la cubierta (techo) para direccionar dichos vientos, e inclusive alejar aquellos desfavorables (norte) o aprovechar los del sur.

Encuestas. Finalizado el ciclo lectivo, se realizó una encuesta a los alumnos, a fin de no incurrir en una visión unipersonal de los resultados, y concibiendo el ciclo de diseño y programación de la enseñanza como un proceso de investigación-acción y la propuesta curricular como hipótesis de trabajo para ser sometida a prueba, desde las perspectivas de SCHWAB J., STENHOUSE L., ELLIOT J. (citados en ARAUJO, 2006), DAY (2005) y SCHÖN (1992). Dichos autores son referentes del Enfoque Práctico de elaboración e indagación del currículum, que recupera el valor del conocimiento práctico que construyen los docentes a lo largo de su carrera, como criterio de actuación en las fases de diseño, puesta en práctica y evaluación del currículum, reflexionando "en, sobre y en relación con la acción". El objetivo de la encuesta fue indagar sobre la percepción de los alumnos acerca de la influencia en su proceso de aprendizaje de la experiencia pedagógica de la que fueron partícipes $y$, de esta manera, identificar los puntos críticos sobre los cuales se debe intervenir, en pos de la mejora de la enseñanza.

\section{CONCLUSIONES Y REFLEXIONES FINALES}

El análisis de los logros alcanzados por los alumnos permitió reflexionar sobre diferentes cuestiones valoradas desde la experiencia docente, y contrastadas con la encuesta realizada. A continuación se exponen los principales hallazgos en torno a las distintas dimensiones mencionadas en los objetivos, destacando fortalezas y debilidades de las distintas instancias del ejercicio.

Apreciación de la metodología de trabajo. Las clases teóricas fueron calificadas como muy claras y concisas como introducción para entender e incorporar estrategias de diseño ambiental a los proyectos de arquitectura. El material didáctico re- 
sultó muy útil como herramienta de trabajo, pues se ajustaba a las consignas del ejercicio. Se destaca la generación de espacios de participación, y las actividades de puesta en común -en la etapa de análisis, catalogada como de suma utilidad y enriquecimiento para los alumnos, que promovió el trabajo grupal- contribuyeron a una mejor interpretación de los contenidos dados en las clases, a partir del análisis que cada grupo realizó. En la mayoría de los casos el trabajo de investigación y análisis de modelos colaboró en la etapa de diseño al surgimiento de la idea, sin ser esta copia de la obra analizada. Asimismo, propició la participación activa, incentivando en los alumnos la comunicación a nivel técnico-expresivo de sus trabajos y el desarrollo de la capacidad de síntesis.

Constituyó un desafío el tener que desenvolverse delante de los compañeros y docentes. Por otra parte, algunos alumnos señalaron que los paneles síntesis no fueron confeccionados muy claramente. Esto abre una nueva línea de acción, en cuanto a la necesidad de brindar apoyatura para la comunicación de las producciones grupales.

En la etapa de diseño creativo, el esquicio de ideas generadoras en maquetas, si bien tuvo cierta resistencia al principio, estimuló a los alumnos a operar el diseño considerando distintas propuestas espaciales, funcionales y tecnológicas, superando la concepción bidimensional que generalmente se resuelve en planta. Además, la elaboración de maquetas de estudio ayudó a superar el "salto al vacio" para el surgimiento de las primeras ideas, sin caer en formalismos, sino con una justificación concreta de cada alternativa. Sumado a ello, permitió verificar en los distintos momentos del proceso de diseño la pertinencia de las estrategias DAC aplicadas, tornándose en una importante herramienta proyectual, más que en objeto de exposición del resultado final. El esquicio fue calificado por los alumnos como una manera rápida y eficaz de transmitir la idea generadora y trabajar sobre ella en las correcciones de una manera muy simple. Sin embargo, hay opiniones diversas referidas a la evolución del proceso de diseño, que señalan dificultades para hallar compatibilidad entre la idea generadora basada en una estrategia DAC y el diseño creativo para el caso del centro de capacitación, a nivel funcional.

Motivación. El planteo de las estrategias DAC, no demasiado habituales en nuestro medio, sumado al compromiso de proponer una solución proyectual original, condujo a los alumnos a la búsqueda y manejo de información dejando de lado la actitud pasiva de receptores de datos. Asimismo, la formulación del tema-problema para resolver impulsó la búsqueda de respuestas alternativas a las convencionales. Desde su rol de alumnos calificaron la experiencia como muy provechosa, por ser el diseño sustentable un tema de interés. Se sintieron motivados en el desarrollo del ejercicio de DAC, por ser algo nuevo. Más que un trabajo práctico, significó un reto, y lograron resolver con éxito el tema-problema.

Desarrollo de la capacidad creativa y el pensamiento crítico e intuitivo. La diversidad de propuestas resultantes demuestra que, cuando existe un aporte sensible y comprometido, basado en el conocimiento científico, la solución tiene un valor agregado, confluyendo en la obra propuesta, valores técnicos, funcionales y estéticos. Los alumnos calificaron el ejercicio como uno de los más interesantes del año, ya que el uso de estrategias y pautas sustentables de alguna manera siempre va a estar ligado a sus proyectos. Lograron afianzar una postura reflexiva, teniendo ahora las herramientas para Ilevarla adelante. Consideraron como fundamental la adecuación climática del producto arquitectónico mediante estrategias de diseño sustentable. Esta experiencia resulta innovadora en lo que respecta al modo de abordaje de la idea generadora de un objeto arquitectónico, partiendo del diseño ambientalmente consciente, sobre la base de una 
"Al haber una suma de personas, con diferentes roles y opiniones, facilita la aparición de una gama más amplia de posibilidades. Siendo un solo proyectista es más difícil no 'casarse' con una idea y desarrollar otras imparcialmente" (LITWIN, SORONDO Y URIBURU, 2008)...".

categoría de análisis estudiada y desarrollada en profundidad y principalmente su materialización como producto original realizado por los alumnos. Recuperación de conocimientos previos e integración de contenidos. Los alumnos señalaron que todo el trayecto del trabajo lo relacionaron con la materia Teoría del Diseño, lo que permitió comprender todo lo que implica proyectar; con Sistemas de Representación y Expresión, en la representación de vegetación y sombras; con Morfología l, en el juego con operaciones de simetría para obtener tipologías interesantes. Lograron además aplicar lo aprendido en los siguientes ejercicios del ciclo lectivo (aprendizaje significativo), y señalaron que la metodología de trabajo implementada los ayudó a obtener resultados en tiempos cada vez menores y con menor cantidad de correcciones.

Predisposición para el trabajo grupal colaborativo como modo para abordar la masividad. El trabajo en modalidad grupal, con consignas que propician la participación de cada integrante del grupo y la interacción de los grupos entre sí, permitió afrontar con éxito la condición de masividad dada por una matrícula anual de 230 alumnos en promedio, a cargo de cuatro docentes de planta permanente. Constituyó una experiencia satisfactoria para la mayoría, a excepción de casos particulares que no lograron una buena compatibilidad e integración. Si bien muchas veces la producción grupal no llega a tener la calidad esperada, siempre permite verificar un producto enriquecido por los aportes de cada integrante y una gran carga conceptual de ideas. "Al haber una suma de personas, con diferentes roles y opiniones, facilita la aparición de una gama más amplia de posibilidades. Siendo un solo proyectista es más difícil no 'casarse' con una idea y desarrollar otras imparcialmente" (LITWIN, SORONDO Y URIBURU, 2008). Al mismo tiempo, se rompe la inercia individualista que impera usualmente en los talleres, lográndose cooperación y colaboración cruzada entre pares, que culmina siendo altamente satisfactoria. "Cuando los estudiantes participan activamente en el trabajo grupal resolviendo tareas intelectualmente atractivas, mientras más conversan y trabajan en conjunto, más aprenden" (LOTAN, R. A.; WHITCOM, J. A., 1999, en COLS, 2004).

Grado de aprendizaje de los contenidos disciplinares. El ejercicio ha ofrecido resultados muy productivos y muy variados, caracterizados por una composición sustentable en su totalidad. Más allá de que se haya dado especial atención a la categoría de estrategias DAC, que se asignó a cada equipo, han logrado propuestas integrales y creativas, destacándose con énfasis la idea generadora de Diseño Ambientalmente Consciente. Al ser exhaustiva la etapa de estudio de la estrategia que les tocó trabajar, lograron plasmarla en una idea generadora interesante y su posterior desarrollo, destacando su utilidad, no solo para los siguientes ejercicios, sino también para su vida profesional. El enfrentarse a problemas los lleva hacia un pensamiento crítico y creativo; recuerdan con mayor facilidad la información, ya que es más significativa para ellos, fomentando que lo aprendido se comprenda y no solo se memorice, logrando así la aplicación de conceptos bioclimáticos en proyectos de arquitectura en distintas escalas, con resolución de unidad y de conjunto edilicio.

Evaluación. En general, la evaluación en las diferentes instancias del ejercicio y la final les pareció correcta, obteniendo la calificación que estaba dentro de sus expectativas. Sin embargo, destacaron que si bien tuvieron un buen proceso, a la hora del desarrollo de la documentación técnica hubo algunas fallas que bajaron el nivel del trabajo, reflejándose en la nota final. Nuevamente se manifiesta la necesidad de brindar apoyatura en representación y expresión.Como propuesta para mejorar el ejercicio, algunos alumnos señalaron la necesidad de individualizarlo, para lograr una mayor preocupación y esmero de cada uno de los alumnos, por la crisis ambiental que vivimos, "porque cuantos más seamos, más rápido y fácil llegaremos hacia una Arquitectura Sustentable". 
Nuevas líneas de acción. Durante el Ciclo Lectivo 2013, encuadrándonos en el contexto pedagógico definido por la Pirámide B, "Problemática del Hábitat en la Región NEA", se continuó desarrollando la propuesta pedagógica descrita, con algunas innovaciones, esta vez mediante el proyecto de una " $V i-$ vienda de Interés Social en Dúplex", en el marco del Plan Procrear, que ha tenido impulso a nivel nacional, además de representar una temática más cercana a la realidad y el contexto en que el alumno se desenvuelve. Se abordó este tema entendiéndose como vivienda de interés social a un tipo de carencia habitacional que a la sociedad le interesa y debe resolver. Esta responsabilidad de solución tiene que ver no solo con aspectos cuantitativos (en términos económicos), sino además con aspectos cualitativos en lo que respecta al mejoramiento de la calidad de vida de los usuarios, a la integración del objeto arquitectónico a su contexto, a la reducción de la demanda de energía convencional y al aprovechamiento de fuentes energéticas alternativas. En los ciclos lectivos anteriores, los alumnos no tuvieron restricciones desde el punto de vista económico, en cuanto a la selección de los materiales y tecnologías constructivas, mientras que para el desarrollo de este ejercicio, partimos de la siguiente problemática: ¿qué aportes se puede realizar a la vivienda de interés social, incorporando criterios de eficiencia energética y buenas condiciones de habitabilidad como valor agregado, sin incrementar el costo final de construcción? La estrecha relación entre las actividades de investigación y transferencia a la docencia adquiere un carácter multiplicador y demostrativo, pues alimenta, informa, verifica y orienta las actividades de enseñanza-aprendizaje con conocimientos científicamente fundamentados, a fin de mejorar la capacidad de los estudiantes y futuros arquitectos, para contribuir eficazmente a un hábitat construido más sustentable en la práctica profesional de la arquitectura.

\section{BIBLIOGRAFÍA}

ARAUJO, S. (2006). Docencia y enseñanza. Una introducción a la didáctica. Buenos Aires: Universidad Nacional de Quilmes. Cap. 2.

BOUTET, M. L. y VIRILI, J. C. (2012). Diseño Ambientalmente Consciente (DAC). Hacia una Arquitectura Sustentable para el Hombre y la Sociedad. "Publicación digital Cátedra Arquitectura II - UPB, Biblioteca Arq. Roberto Champion, FAU - UNNE. Resistencia.

ITESM - Instituto Tecnológico y de Estudios Superiores de Monterrey (s. f.). Dirección de Investigación y Desarrollo Educativo, Vicerrectoría Académica. Las Estrategias y Técnicas Didácticas en el Rediseño. El método de proyectos como técnica didáctica. Recuperado el 28/06/14 de http://www.mineducacion.gov.co/1621/article-238238.html.

COLS, E. (2004). Programación de la enseñanza. Ficha de cátedra Didáctica I. Buenos Aires: UBA. Facultad de Filosofía y Letras.

COLS, E. (2008). Las estrategias de enseñanza en la universidad. Conferencia abierta. "Especialización en Docencia Universitaria". Facultad de Humanidades, UNNE.

CZAJKOWSKI, J. y GÓMEZ, A. (2009). Arquitectura sustentable. Ed. Clarín. Buenos Aires.

DAY C. (2005). Formar docentes. Cómo, cuándo y en qué condiciones aprende el profesorado. Narcea. Madrid.

LITWIN, B.; SORONDO, R.; URIBURU, J. (2008). Pasos hacia una Metodología de Diseño, Ed. Nobuko, Buenos Aires, Argentina.

ONU - Organización de las Naciones Unidas (1987). Our Common Future. Reporte de la Comisión Mundial de Ambiente y Desarrollo.

SCHON, D. (1992). La formación de profesionales reflexivos. "Hacia un nuevo diseño de la enseñanza y el aprendizaje en las profesiones". España: Paidó 